\title{
The NPR1 ortholog PhaNPR1 is required for the induction of PhaPR1 in Phalaenopsis aphrodite
}

\author{
Jen-Chih Chen ${ }^{1,2}$, Hsiang-Chia Lu ${ }^{3}$, Cheng-En Chen ${ }^{3}$, Hua-Fang Hsu ${ }^{3}$, Hong-Hwa Chen ${ }^{5,6,7}$ and Hsin-Hung Yeh ${ }^{3,4^{*}}$
}

\begin{abstract}
Background: Systematic acquired resistance (SAR) is an effective broad-spectrum defense mechanism that confers long-lasting protection against biotrophic pathogens trough defense related salicylic acid (SA) signaling. Gene(s) involved in SAR have been extensively studied in dicot plants; however, remains largely unresolved in monocot plants. NPR1, an evolutionary conserved gene, plays a central role in SAR, and PR-1 is widely used as a marker for effective SA signaling.

Results: We identified NPR1 and PR-1 homologous genes, PhaNPR1 and PhaPR1, from an economically important orchid, Phalaenopsis aphrodite, and characterized their roles in SA signaling and Cymbidium mosaic virus (CymMV) resistance. A phylogenetic analysis of NPR1 homologs showed that these genes appear to have evolved before angiospermy. Similar to Arabidopsis NPR1, PhaNPR1 was only moderately induced upon SA treatment and CymMV infection. Although PhaPR1 shows only 36\% identity with AtPR1, its promoter shared conserved elements with those of other PR-1 genes, and it was induced upon SA treatment and CymMV infection. After CymMV infection, silencing on PhaNPR1 also reduced PhaPR1 expression; however, CymMV accumulation was not affected.
\end{abstract}

Conclusions: In conclusion, after virus infection, PhaNPR1 is required for PhaPR1 induction, but plays little role in defense against CymMV.

Keywords: NPR1; PR1; Phalaenopsis aphrodite; Salicylic acid; Systemic acquired resistance

\section{Background}

Orchidaceae is a widespread monocot family that undergoes rapid speciation. More than 20,000 species in 850 genera have been recorded, and it is believed to be the largest family of angiosperms. Due to adaptive radiation, orchids have evolved an array of strategies to successfully colonize diverse terrestrial ecosystems. Therefore, they provide rich resources to study evolution and the mechanisms of plant-environment interactions. Their diverse flower colors and shapes make them attractive in the floral industry. Orchids are economically important in countries worldwide; however, their production is harmed by pathogen attacks. Our knowledge concerning orchid defense is still limited.

\footnotetext{
* Correspondence: hyeh@ntu.edu.tw

${ }^{3}$ Department of Plant Pathology and Microbiology, National Taiwan

University, 1, sec 4, Rooselvet Road, Taipei 106, Taiwan

${ }^{4}$ Research Center for Plant Medicine, National Taiwan University, Taipei 106,

Taiwan

Full list of author information is available at the end of the article
}

Plants rely on innate immunity to counteract challenges from pathogens. The defense system is tightly regulated and coordinated through several inducible responses involving phytohormones, such as salicylic acid (SA), jasmonic acid (JA), and ethylene (Pieterse et al., 2009). SA is important for resistance against biotrophic pathogens by inducing systemic acquired resistance (SAR), an effective broad-spectrum defense mechanism that confers long-lasting protection. When SAR occurs, several pathogenesis-related genes ( $P R$ genes) are induced locally at the site of infection and systemically in distal plant tissues (Durrant and Dong, 2004). Studies have demonstrated that many PR proteins have antimicrobial properties (van Loon et al., 2006); however, a single $P R$ gene often confers limited resistance to pathogen invasion in transgenic plants. Thus, it is generally believed that the concerted expression of many $P R$ genes confers SAR resistance (Durrant and Dong, 2004).

The PR-1 gene family was the first identified among a host of genes involved in plant defense against pathogens, including oomycetes and fungi. PR-1 proteins are 
evolutionary conserved among plants, fungi and animals; however, the precise biological functions of PR-1 proteins remain elusive (van Loon et al., 2006). In addition, the expression of $P R-1$ genes has been used as a molecular marker to monitor SA signaling and the onset of SAR in various plants, including Arabidopsis, tobacco, tomato, rice and barley (van Loon et al., 2006). In Arabidopsis, 22 PR-1 paralogs have been identified; however, only one, AtPR-1 (AT2G14610), is induced by SA, and it has been suggested to be the lone PR-1 protein for induced resistance (van Loon et al., 2006). Unlike Arabidopsis, 12 rice PR-1 paralogs were induced upon pathogen attack (Mitsuhara et al., 2008). The regulation of different $P R$ 1 genes can be diverse; therefore, it is difficult to predict $P R-1$ regulation based on sequence similarity.

The induction of AtPR-1 and other $P R$ genes during SAR relies on the expression of a functional NPR1 (nonexpressor of pathogenesis-related genes 1), which is a conserved central positive regulator of SA signaling (Durrant and Dong, 2004). Recently, NPR1 was found to serve as a receptor for SA through $\mathrm{Cys}^{521 / 529}$ in Arabidopsis (Wu et al., 2012). Mutations in NPR1 result in breached local basal resistance and a higher accumulation of virulent pathogens, such as Pseudomonas syringae pv. maculicola (Glazebrook et al., 1996). In contrast, overexpression of NPR1 protein enhances broad-spectrum disease resistance in Arabidopsis, rice and wheat, suggesting that the NPR1mediated defense mechanism is evolutionary conserved across a wide range of species (Cao et al., 1998; Chern et al., 2001; Makandar et al., 2006). In addition, NPR1 homologs from rice (OsNPR1/NH1), Theobroma cacao (Tc NPR1), or Vitis vinifera (VvNPR1.1) were able to complement an npr1 mutation in Arabidopsis (Le Henanff et al., 2011; Shi et al., 2010). However, in contrast to NPR1 overexpression in Arabidopsis, the overexpression of OsNPR1/ $\mathrm{NH1}$ in rice spontaneously activated resistant genes and resulted in a lesion-mimic phenotype (Chern et al., 2005). This result indicates regulation diversities in SAR among different species.

In Arabidopsis, the transcription of NPR1 is only moderately induced upon SA treatment, and post-translational regulation plays a key role in NPR1 activation (Durrant and Dong, 2004). In the uninduced state, NPR1 is present as an oligomer in the cytosol; however, the induction of SAR changes the cellular redox potential of NPR1 and results in its reduction to a monomeric form (Durrant and Dong, 2004). This event results in the accumulation of NPR1 in the nucleus, which interacts with the TGA family of basic leucine zipper (bZIP) transcription factors. The NPR1-TGA complex subsequently induces the expression of defenserelated genes, including the $P R$ genes (Durrant and Dong, 2004). The cysteine residues Cys ${ }^{82}$ and $\mathrm{Cys}^{216}$ in the NPR 1 protein are important for the oligomer formation, and the mutation of $\mathrm{Cys}^{150}$, Cys ${ }^{155}$, or $\mathrm{Cys}^{160}$ leads to a reduction of
NPR1 accumulation (Durrant and Dong, 2004). These results indicate that the conserved cysteine residues are important for its regulation at the protein level. Recently, it was shown that the turnover of NPR1 plays dual roles to both prevent and stimulate gene transcription in the regulation of plant immunity (Spoel et al., 2009).

In this study, we aimed to identify key components of SAR, NPR1 and PR1, from a commercially important orchid, Phalaenopsis aphrodite subsp. formosana and to understand their roles in SA signaling as well as virus defense. Phylogenetic analyses revealed that PhaNPR1 is an ortholog of NPR1 while PhaPR1 protein shares only moderate similarity with known PR1-like proteins. In spite of low sequence similarity of PhaPR1 to other PR1-like proteins, PhaPR1 was strongly induced upon SA treatment and virus infection but not JA treatment. The transient knockdown of the PhaNPR1 suggested that PhaNPR1 may act upstream of PhaPR1 though defense against CymMV may not require its action. This work provides the basis for further studies of SAR in orchids.

\section{Methods}

\section{Growth conditions and chemical applications}

Phalaenopsis aphrodite subsp. formosana plants, a commercial orchid variety, were purchased from the Taiwan Sugar Research Institute (Tainan, Taiwan). The plants were maintained in an insect-proof controlled greenhouse with a $12 \mathrm{~h}$ photoperiod $\left(200 \mu\right.$ mole $\left.\mathrm{m}^{-2} \mathrm{~s}^{-1}\right)$ at $25^{\circ} \mathrm{C}$. Upon receipt, reverse transcription-polymerase chain reaction (RT-PCR) was conducted to ensure that the orchid plants used were free of virus. The primer pairs, ORSV-CP-F/ORSV-CP-R and CymMV-CP-F/CymMV-CP-R, targeting the coating proteins of two prevalent orchid viruses, ORSV and Cym MV, were used (Additional file 1: Table S3). For phytohormone treatment, sodium salicylate (Sigma, $10 \mathrm{mM}$ in water) and methyl jasmonate (Sigma, $45 \mathrm{mM}$ in $1 \%$ [v/v] ethanol) were directly sprayed onto the plants. The control plants were sprayed with water or $1 \%$ ethanol.

\section{Identification of PhaNPR1 and PhaPR1}

The rapid amplification of cDNA ends (RACE) was performed to obtain $5^{\prime}$ and 3 '-end cDNAs of the NPR1 and PR-1 homologs PhaNPR1 and PhaPR1 from P. aphrodite subsp. formosana using the SMART-RACE cDNA amplification kit (Clontech; Mountain View, CA, USA) according to the manufacturer's instructions. The primers used for this study are described in Additional file 1: Table S3. The primer pairs, NPR1F/NPR1R were designed from the NPR1 conserved region to amplify partial PhaNPR1 cDNA using RNA isolated from $P$. aphrodite subsp. formosana treated with salicylic acid. The amplified fragment was cloned, and the complete sequences were determined. NP R1R was used as a gene-specific primer (GSP) for $1^{\text {st }}$ RACE PCR and NPR1 $5^{\prime}$ NGSP was used as a $2^{\text {nd }}$ GSP for 
nested PCR to obtain the $5^{\prime}$ end of PhaNPR. The $3^{\prime}$ end of PhaNPR1 was amplified using NPR1 F and NPR13' NGSP as $1^{\text {st }}$ and $2^{\text {nd }}$ GSPs for 3'RACE to amplify the 3' end of PhaNPR1. Both RACE amplified 5' and 3' ends of PhaNPR1 were cloned, and the complete sequences were determined. The primer pairs NPR1 ORFF/NPR1 ORFR were designed from the obtained $5{ }^{\prime}$ - and 3 ' - RACE amplified fragments and used in a PCR reaction to obtain the full-length open reading frame (ORF) of PhaNPR1. The full-length ORF of PhaNPR1 was cloned, and the complete sequence was determined. The cloning of PhaPR1 was performed using essentially the same method as that used to clone PhaNPR1, except the primer pair PR1F/PR1R were used in the PCR reaction to obtain partial PhaPR1 cDNA, PR1 5' GSP and PR1 5' NGSP primers were used as $1^{\text {st }}$ and $2^{\text {nd }}$ GSPs in the $5^{\prime}-$ RACE reaction, PR1 3' GSP and PR1 $3^{\prime}$ NGSP primers were used as $1^{\text {st }}$ and $2^{\text {nd }}$ GSPs in the $3^{\prime}$-RACE reaction, and the entire PR1 ORF was amplified using the primer pair PR1 ORFF/PR1 ORFR.

\section{Genomic DNA extraction}

Genomic DNA from P. aphrodite was purified as previously described (Carlson et al., 1991) with some modifications. Two grams of homogenized leaf tissue were treated with $15 \mathrm{ml}$ of $65^{\circ} \mathrm{C}$ pre-warmed extraction buffer (100 mM Tris-HCl, 1.4 M NaCl, 20 mM EDTA, 2\% CTAB, 1\% PVP, $200 \mu \mathrm{l} \beta$-mercaptoethanol) and incubated for $2 \mathrm{~h}$ at $65^{\circ} \mathrm{C}$. Subsequently, $15 \mathrm{ml}$ of chloroform was added into the extraction mix, and the extraction mixture was mixed at room temperature for $15 \mathrm{~min}$; the mixture was centrifuged at 5,000 $\times \mathrm{g}$ for $15 \mathrm{~min}$ at $4^{\circ} \mathrm{C}$. The aqueous phase was filtered through a $70 \mu \mathrm{m}$ cell strainer (BD Biosciences; Bedford, MA, USA), and the DNA was precipitated using isopropanol. The precipitated DNA was dissolved in $1.3 \mathrm{ml}$ of $1 \mathrm{M} \mathrm{NaCl}$, and treated with RNase to remove the remaining RNA. After RNase treatment, a phenol-chloroform extraction step followed by isopropanol precipitation was conducted to obtain purified DNA.

\section{Cloning of PhaPR1 Promoter}

The 5 '-flanking region of PhaPR1 was amplified using the GenomeWalker ${ }^{\text {rat }}$ Universal kit (Clontech). The genomic DNA from $P$. aphrodite was digested with restriction enzymes, Dra I, EcoR V, Pvu II and Stu I, to generate bluntend fragments, and subsequently the GenomeWalker Adaptors were ligated to the DNA fragments to generated four DNA libraries. The ligated products were used as templates, and the primer pair, Adaptor Primer 1/PR1GSP1 (Additional file 1: Table S3), was used in the primary PCR reaction. A nested $\mathrm{PCR}$ reaction using the primer pair, Adaptor Primer 2/PR1GSP2 (Additional file 1: Table S3), was performed to identify the gene-specific 5 '-flanking sequence. The PCR products were cloned into a pGEM-T Easy vector (Promega; Madison, WI, USA) and sequenced.

\section{Infection with Cymbidium mosaic virus}

A total of $0.5 \mathrm{~g}$ of CymMV-infected plant tissue was ground in $300 \mu \mathrm{l}$ of inoculation buffer $\left(0.05 \mathrm{M} \mathrm{NaH}_{2} \mathrm{PO}_{4} / \mathrm{Na}_{2} \mathrm{HPO}_{4}\right.$ $\mathrm{pH}$ 7.0) and manually rubbed into carborundum-dusted Phalaenopsis leaves with hands wearing latex gloves. The inoculated leaves were washed with excess distilled water.

\section{Construction of gene silencing vectors and infiltration with Agrobacteria}

The oligonucleotide pairs PhaNPR1-hpRNA-F1/PhaNPR1hpRNA-R1 and PhaNPR1-hpRNA-F2/PhaNPR1-hpRNAR2 (Additional file 1: Table S3) were used to obtain the PhaNPR1 short hairpin fragments PhaNPR1-hpRNA-1 and PhaNPR1-hpRNA-2, respectively. Each set of primer pairs was mixed, denatured at $72^{\circ} \mathrm{C}$ for $10 \mathrm{~min}$, and annealed at $25^{\circ} \mathrm{C}$ for $10 \mathrm{~min}$. Both resulting double-stranded fragments were cloned into the Gateway entry vector $\mathrm{pENTR}^{\mathrm{m}} /{ }^{\mathrm{D}}-\mathrm{TOPO}^{\circ}$ (Invitrogen; Carlsbad, CA, USA) followed by an LR Gateway cloning reaction (Invitrogen) to transfer the fragments into pB7GWIWG2(I) to obtain pB7G-NPR1-1 and pB7G-NPR1- 2. The constructs were transformed into Agrobacterium tumefaciens LBA4404 by electroporation. The verified $A$. tumefaciens strains were cultured in $2 \mathrm{ml}$ of YEB medium $(5 \mathrm{~g} / \mathrm{L}$ beef extract, $1 \mathrm{~g} / \mathrm{L}$ yeast extract, $5 \mathrm{~g} / \mathrm{L}$ peptone, $5 \mathrm{~g} / \mathrm{L}$ sucrose, $0.5 \mathrm{~g} / \mathrm{L} \mathrm{MgCl}_{2}$ ) containing $100 \mathrm{mg} / \mathrm{L}$ kanamycin and $100 \mu \mathrm{M}$ acetosyringone and grown at $28^{\circ} \mathrm{C}$ for overnight. The next day, 1 $\mathrm{ml}$ of the bacterial culture was transferred into $10 \mathrm{ml}$ of YEB medium containing $100 \mathrm{mg} / \mathrm{L}$ kanamycin and $100 \mu \mathrm{M}$ acetosyringone and further incubated at $28^{\circ} \mathrm{C}$ until reaching an $\mathrm{OD}_{600}$ of 1.0 1.2. The A. tumefaciens cultures were centrifuged at $3,000 \times \mathrm{g}$ for $10 \mathrm{~min}$, and the cells were resuspended in $1 \mathrm{ml}$ of infiltration medium (10 mM MES, $10 \mathrm{mM} \mathrm{MgCl}_{2}$, and $100 \mu \mathrm{M}$ acetosyringone) and incubated at room temperature for $3 \mathrm{~h}$. Each Phalaenopsis plant was infiltrated with $100 \mu \mathrm{l}$ of $A$. tumefaciens suspension.

\section{RNA isolation, real-time RT-PCR}

For RT-PCR, total RNA was extracted from the orchid plants as described (Tian et al., 1996). The RNA was treated with RNase-free DNase (Ambion) to eliminate genomic DNA contamination. Subsequently, $0.5 \mu \mathrm{g}$ of DNA-free RNA from each sample was used for the synthesis of first strand cDNA using Moloney murine leukemia virus (MMLV) reverse transcriptase according to the manufacturer's instructions (Invitrogen). For real-time RT-PCR, $200 \mathrm{ng}$ total RNA treated with TURBO DNA-free kit (Ambion) was used as a template for CDNA synthesis using Moloney murine leukemia virus (MMLV) reverse transcriptase following the manufacturer's instructions (Promega). The cDNA corresponding to 50 ng total RNA was used for realtime PCR using a SYBR Green staining method (ABI StepOne $^{\text {mix }}$ Real-Time PCR system, Applied Biosystems). The primers used are listed in Additional file 1: Table S3. 
PCR products of analyzed genes were sequenced to validate the correct analysis of gene targets. Genes analyzed were from 3 biological replicates and each sample was analyzed for 3 technical replicates. The relative quantification was calculated according to the manufacturer's instructions (Applied Biosystems). The Ubiquitin 10 gene was used as an internal quantification control.

\section{Phylogenetic analysis}

Phylogenetic analysis of the NPR1 genes was conducted using MAGA 5.0 with the maximum likelihood (ML) method. The branch support was estimated using bootstrapping with 1,000 replicates. The sequences used in this study were obtained from NCBI GenBank and are listed in the supplementary information.

\section{Results}

Sequence analysis of PhaNPR1 and PhaPR1

To identify NPR1 and PR1 homologs in Phalaenopsis aphrodite subsp. formosana, primers were designed based on the conserved domain sequences to amplify partial sequences of NPR1 and PR1. Subsequently, RACE-PCR was used to obtain full-length transcripts of both genes. The NPR1 homolog PhaNPR1 (GenBank accession no. JN630802) was identified, which comprises a full-length 1938 bp sequence encoding a 546 amino acid protein with a predicted molecular weight of $60.9 \mathrm{kDa}$ and pI of 5.4. The protein shows $50 \%$ sequence identity with the Arabidopsis NPR1 (AT1G64280) and 61\% identity with the rice NPR1 (Os01g0194300). The protein also shared conserved domains with other known NPR homologs: a BTB/POZ domain (amino acids 56 to 177), ankyrin repeat domains (amino acids 238 to 369), and a NPR1/NIM1 like defense protein $C$ terminal motif (amino acids 353 to 546) (Figure 1A). A multiple alignment using ClustalX showed that the conserved cysteine residues, including the residues corresponding to AtNPR $1 \mathrm{Cys}^{82}$ and $\mathrm{Cys}^{216}$, which are required for oligomerization, were also present in PhaNPR1 (Figure 1B). In addition, both motifs required for NIM1-INTERACTING (NIMIN) protein binding, the LENRV motif and a NIMIN binding site, were found in PhaNPR1 (Figure 1B). A phylogenetic analysis of NPR1
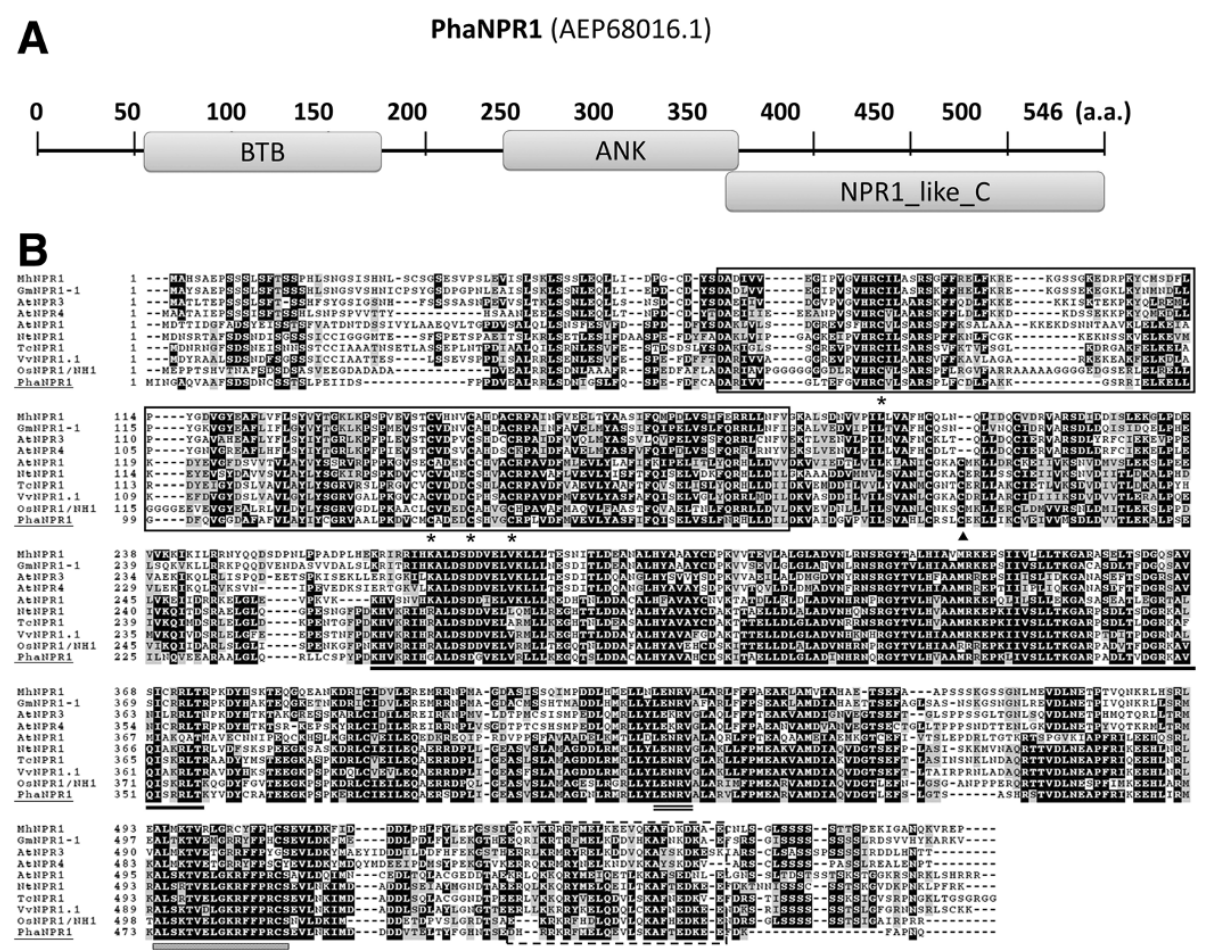

Figure 1 Protein structures of Phalaenopsis NPR1. (A) A schematic representation of PhaNPR1. The three major domains, BTB, ankyrin repeats domains (ANK), and C terminal domain (NPR1_like_C), of this protein are indicated with boxes. The two 21-nt regions indicate the regions used for PhaNPR1 silencing. (B) Alignment of PhaNPR1 (underlined) with NPR1 homologs whose localizations have been determined. AtNPR3, AtNPR4 from Arabidopsis (Liu et al., 2005; Zhang et al., 2006), MhNPR1 from Malus hupehensis (Zhang et al., 2011), GmNPR1-1 from Glycine max (Sandhu et al., 2009), VvNPR1.1 from grapevine (Le Henanff et al., 2011), and NtNPR1 from tobacco (Maier et al., 2010), were localized to the nucleus, while AtNPR1 from Arabidopsis, TcNPR1 from Theobroma cacao (Shi et al., 2010), and OsNPR1/NH1 from rice were localized to the cytoplasm without reductants. The BTB/POZ and ANK domains, and the C-terminal nuclear localization signal (NLS) are indicated with a solid-lined box, underline, and dashed-lined box, respectively. The conserved LENRV motif is indicated with a double line and a NIMIN binding site is labeled with (). The conserved cysteine residues are indicated with an asterisk (*), and the cysteine residue corresponding to $\mathrm{C}^{216}$ in Arabidopsis, which is important for the redox regulation of NPR1, is indicated with a black triangle. 
proteins using a bootstrap consensus for maximum likelihood (ML) revealed that NPR1 homologs can be classified into three major groups, NPR1, NPR3, and BOP, with high confidence in which both dicot and monocot species have paralogs in each major group (Figure 2). The phylogenetic analysis also indicated that members of the NPR1 and NPR3 gene families are closely related, and might have evolved from an NPR ancestor after the divergence of the $N P R$ and $B O P$ genes prior to the evolution of angiospermy (Figure 2). We therefore proposed the classification of NPR homologs into NPR and BOP clades; the NPR clade is further divided into two NPR1 and NPR3 subclades. PhaNPR1 was clearly grouped into the NPR1 subclade with other members from monocot plants, including Oryza and Musa acuminata (Figure 2).

The PR1 homolog, PhaPR1 (GenBank accession no. JX137044), is 767 bp sequence encoding a 169 amino acid protein containing a 21 amino acid signal peptide, as predicted by SignalP, and a SCP domain, which is a conserved domain present in most PR1-like proteins. The predicted molecular weight and $\mathrm{pI}$ of the mature protein are $16.2 \mathrm{kDa}$ and 6.95, respectively. The conserved features, which include four $\alpha$-helices, four $\beta$-strands and six conserved cysteine residues, were present in PhaPR1 (Figure 3). However, this protein only shares moderate sequence identities with PR-1 proteins with antimicrobial activity. The identities to

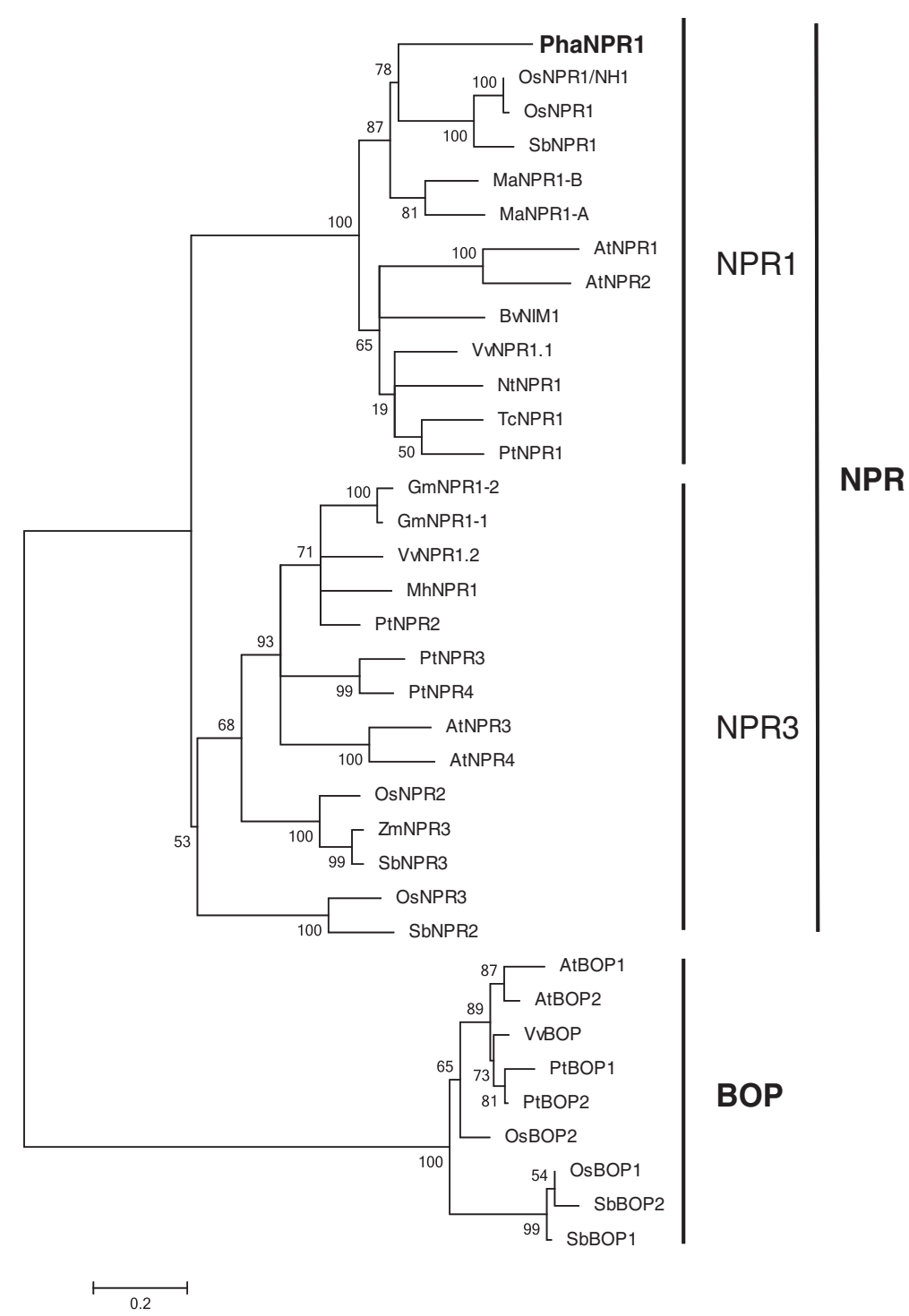

Figure 2 Phylogenetic tree of the selected NPR1 homologs. The deduced amino acid sequences of NPR1 homologs from different plant species were used to construct the tree using the maximum likelihood method with 1000 bootstrap values indicated. The information for the homologs used in the analysis is listed in Additional file 1: Table S2. PhaNPR1 is indicated in bold. Members from the five species with reference genomes were included to identify orthologous groups. Three orthologous groups were identified and named NPR1, NPR3, and BOP. Because the NPR1 and NPR3 groups were closely related, these two groups were joined to form a larger NPR group. 


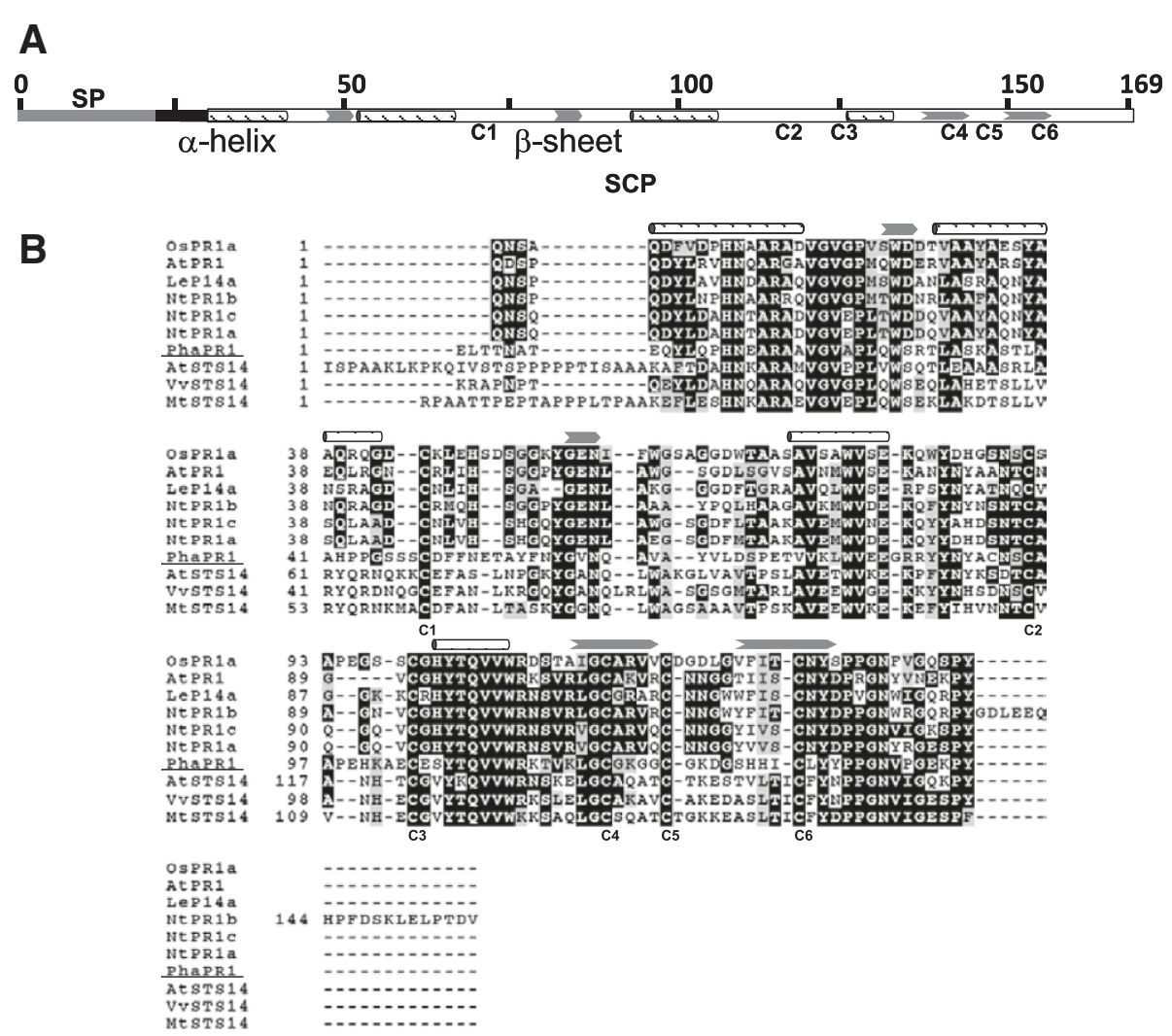

Figure 3 The structure of Phalaenopsis PR1. (A) A schematic diagram of PhaPR1. The full-length, 169 amino acid protein contains a predicted signal peptide (SP) of 21 residues in length indicated in gray, the 4 a-helixes are indicated ( $\mathbf{B}$ ), and the $4 \beta$-sheets are also shown ( $)$ ). The 6 conserved cysteine residues are also indicated with C1-C6. (B) Alignment of PhaPR1 (underlined) with PR-1-like proteins, which have antimicrobial functions, and STS14-like proteins. The mature protein sequences were used for the analysis. Their accession numbers are OsPR1a (NP_001058815), AtPR1 (AT2G14610), LeP14a (NP_001234314), NtPR1a (CAA31233), NtPR1b (CAA47374), NtPR1c (CAA35666), AtSTS14 (AT5G66590), VvSTS14 (XP_003635089), and MtSTS14 (XP_003611867).

tomato P14a (NP_001234314), Arabidopsis PR1 (AT2G 14610), rice PR-1a (Os07g0129200), and tobacco PR-1a (CAA31233) are 40\%, 36\%, 35\%, and 35\%, respectively. PhaPR1 is closely related to STS14-like proteins $(\sim 47 \%$ identity), which were first identified in the potato and highly expressed in the pistil (Van Eldik et al., 1996). However, the function of these proteins has not been characterized. PhaPR1 is most similar to a PR-1 like protein in Arabidopsis, AtSTS14 (AT5G66590), with 43\% identity. Although detailed characterizations of these proteins have not been attempted, a search for microarray studies on these genes using Genevestigator revealed that AT5G66590 was induced upon infection of the fungal pathogen Alternaria brassicicola (Additional file 1: Table S1).

\section{Analysis of cis-acting elements in the PhaPR1 promoter}

A $2.5-\mathrm{kb}$ upstream region from the translation start site of PhaPR1 was identified within the genomic DNA of $P$. aphrodite using genome walking. The sequence was analyzed using web-based cis-acting element analysis programs, such as PLACE and PlantCARE. The typical CAAT and TATA boxes are present within $150 \mathrm{bp}$ upstream of the ATG start codon in the promoter region. We identified conserved elements within the 5 '-end sequence flanking the promoter region in PhaPR1 and AtPR1 (Table 1). The 815-bp upstream promoter region of AtPR1 is required for the induction response upon SA treatment (Lebel et al., 1998). In this region, several conserved motifs were identified, including a NF- $\mathrm{B}$ binding motif (LS10), an ATAT TCTT motif (LS9), which was also identified in tobacco $P R-1 a$ and PR-2d promoters, a bZIP transcription factor binding motif (LS7), and a zinc-finger motif (LS4). The LS10 and LS7 motifs are essential for SAR-mediated AtPR1 regulation (Lebel et al., 1998). In the PhaPR1 promoter region, a CGGCATTTCC motif, which is similar to LS10 (GGACTTTTC), at position -440 to -430 , a LS9 motif at position -1125 to -1117 , and two LS4 motifs at positions -647 to -641 and -1011 to -1005 were identified; however, the LS7 motif was not located within the promoter region (Table 1). We did find an ASF1 motif (TGACG) at positions -294 to -289 . This motif was shown to mediate auxin- and salicylic acid-inducible transcription 
Table 1 Conserved sequence of various motifs in the PhaPR1 promoter region

\begin{tabular}{|c|c|c|c|c|}
\hline Motif & Consensus sequence & Sequence in PhaPR1 promoter & Position & Reference \\
\hline LS9 & ATATTCTT & ATATTCTT & -1125 to -1117 & (Lebel et al., 1998) \\
\hline GT-1 & GAAAAA & GAAAAA & -1096 to -1091 & (Park et al., 2004) \\
\hline SEBF & PyTGTCNC & GCTGTCAC & -636 to -630 & (Boyle and Brisson, 2001) \\
\hline \multirow[t]{3}{*}{ W-box } & $(\mathrm{T})(\mathrm{T}) \mathrm{TGACY}$ & TTGACT & -1011 to -1006 & (Rushton et al., 1996) \\
\hline & & TTGACT & -648 to -642 & \\
\hline & & TTGACC & -459 to -454 & \\
\hline$N F-k B$ & GGGACTITCC & CGGCATTTCC & -440 to -430 & (Baeuerle and Baltimore, 1996) \\
\hline ASF1 & TGACG & TGACG & -294 to -289 & (Katagiri et al., 1989) \\
\hline
\end{tabular}

in tobacco TGA transcription factors (Niggeweg et al., 2000). Other consensus motifs identified within the promoter region include PyTGTCNC and several W-box motifs (Table 1). The PyTGTCNC motif has been identified in the promoter region of many $P R$ genes, and it is the binding site of the transcription repressor silencing element-binding factor (SEBF) (Boyle and Brisson, 2001). The W-box is a binding motif that has been identified in WRKY transcription factors. The motif is located within the promoter region of many $P R$ genes and was also identified in the promoter region of the Arabidopsis NPR1 gene; this motif is important for the induction of these genes (Yu et al., 2001).
Virus infection and treatment with SA, but not JA, induce the expression of PhaNPR1 and PhaPR1

Although NPR1 transcripts are only moderately induced upon SA treatment in Arabidopsis, the regulation of SA signaling through NPR1 can be different among different species. We determined whether SA treatment could affect the expression of PhaNPR1. Similar to Arabidopsis NPR1, the PhaNPR1 transcript was detected in healthy untreated plants, and moderately induced after treatment with SA and CymMV inoculation for $24 \mathrm{~h}$ (Figure 4A). A time course of the increased PhaNPR1 expression under SA treatment and CymMV was subsequently conducted, and the accumulation of PhaNPR1 transcripts was detected
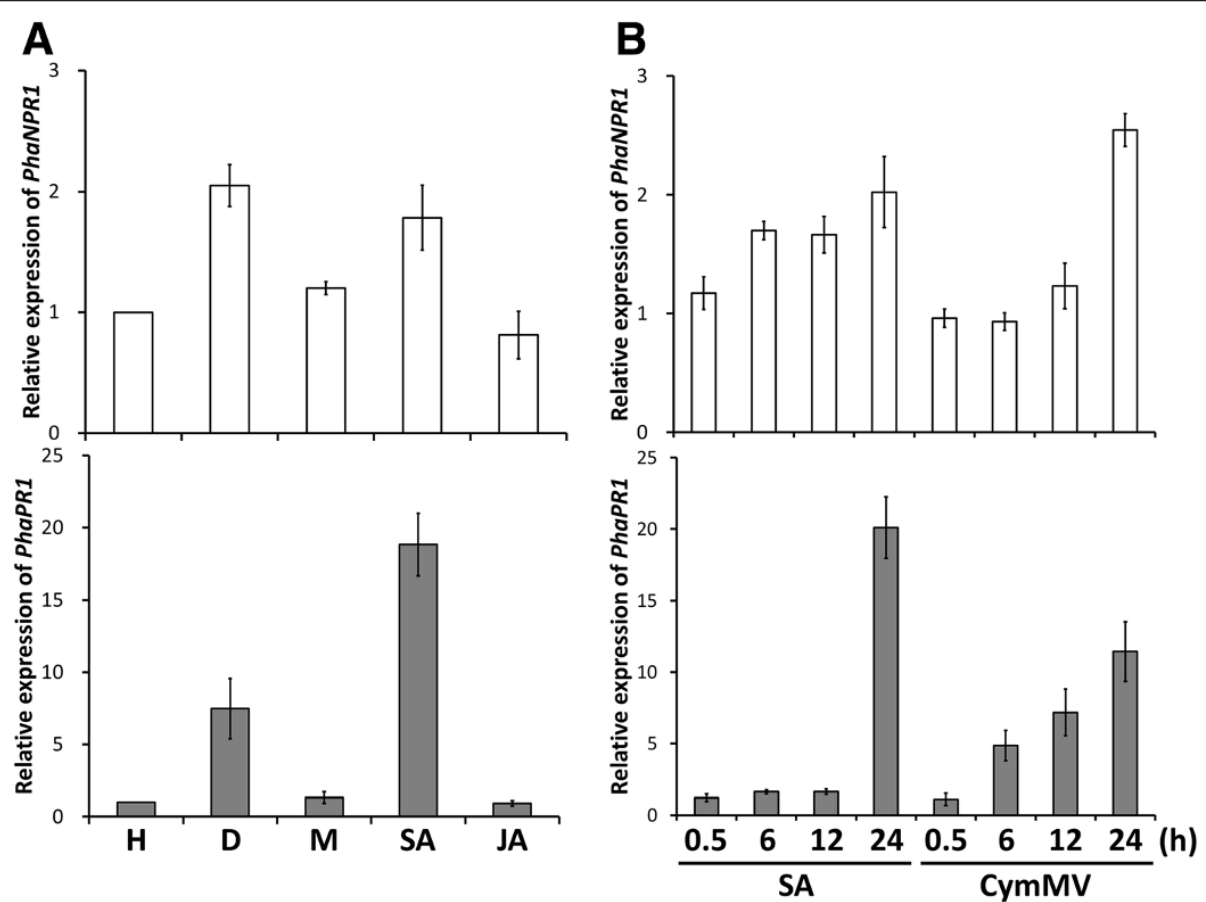

Figure 4 Changes in the expression of PhaNPR1 and PhaPR1 under hormone treatments or virus infection. (A) Analyses of PhaNPR1 and PhaPR1 expression in Phalaenopsis aphrodite subsp. formosana leaves after treatment: (H) Healthy, (D) CymMV infection, (M) 1\% [V/V] ethanol, (SA) Sodium salicylate and (JA) methyl jasmonate. (B) Analyses of PhaNPR1 and PhaPR1 expression at various time intervals after SA treatment or CymMV infection. Data are the mean \pm SD of 3 biological replicates. Expression fold changes are relative to that of healthy controls with UBIQUITIN level as an internal reference. h: treatment hours. 
after $6 \mathrm{~h}$ of SA treatment but was delayed for $6 \mathrm{~h}$ for CymMV inoculated leaves, and the transcript levels of both groups reached to $\sim 2$ fold of the control level after $24 \mathrm{~h}$. Treatment with SA and CymMV infection also induced the accumulation of PhaPR1 transcripts in a manner similar to that of PhaNPR1 (Figure 4). Notably, treatment with JA did not induce the accumulation of PhaNPR1 and PhaPR1 transcripts (Figure 4A).

\section{Knockdown of PhaNPR1 reduces the accumulation of PhaPR1 transcripts but does not affect CymMV concentration}

Our results suggested that NPR1 may play an important role in SA-mediated resistance, and both PhaNPR1 and PhaPR1 are induced upon SA treatment and virus infection; therefore, we transiently knocked down the expression of PhaNPR1 using short hairpin sequences to examine whether it is required for PhaPR 1 transcript accumulation during virus infection and resistance against CymMV. Indeed, after CymMV infected leaves of $P$. aphrodite were transiently inoculated for seven days with Agrobacteria containing a PhaNPR1 short hairpin sequence, the transcript abundance of PhaNPR1 was suppressed, and coincidentally, the transcript level of PhaPR1 was also reduced, especially when using the hpNPR1-2 fragment (Figure 5). However, the suppression of PhaNPR1 expression did not result in over-accumulation of CymMV (Figure 5).

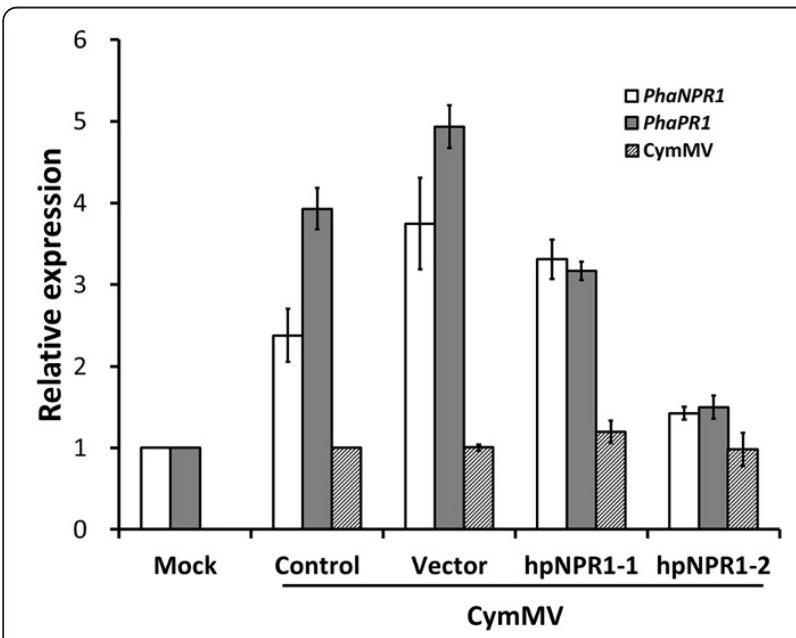

Figure 5 Changes in PhaPR1 expression and CymMV accumulation when PhaNPR1 is silenced. PhaNPR1 expression was transiently knocked down in the leaves of $P$. aphrodite subsp. formosana using Agrobacteria carrying short hairpin constructs. The two constructs used carry hpNPR1-1 and hpNPR1-2, respectively, and the leaves of the control group were only with CymMV infection and vector groups were inoculated with Agrobacteria carrying the empty vector. The mock treatment was inoculation of $1 \%[\mathrm{~V} / \mathrm{V}]$ ethanol. The RNA samples were collected at seven days post inoculation. Data are the mean \pm SD of 3 biological replicates. Expression fold changes are relative to that of the mock treatment for PhaPR1 and PhaNPR1, and to that of the control for CymMV concentration with UBIQUITIN level as an internal reference.

\section{Discussion}

Using PCR, we isolated the full-length cDNA of NPR1 and PR1 homologs from a commercially important orchid, Phalaenopsis aphrodite subsp. formosana. We also identified the promoter sequence of the isolated $P R 1$ gene, PhaPR1. We obtained several interesting findings from the characterization of both genes. First, the phylogenetic analysis of NPR1 homologs from various monocot and dicot species revealed that NPR1 evolved prior to the development of angiospermy, and currently, there are three major groups of NPR1 homologs in every angiosperm species examined thus far (Figure 2). The three phylogenetic groups of Arabidopsis NPR1-like proteins share two conserved domains, BTB/POZ and ankyrin repeats, which are responsible for protein-protein interactions; however, BOP1 and BOP2 lack a conserved nuclear localization signal and have shorter $C$ termini (Hepworth et al., 2005). In Arabidopsis, proteins in the NPR clade have been demonstrated to be important in plant defense, while proteins in the BOP clade are responsible for plant morphogenesis (Cao et al., 1997; Hepworth et al., 2005; Liu et al., 2005; Zhang et al., 2006). However, all NPR1 paralogs might also share redundant functions in SA perception. The growth of plants can be suppressed through treatment with benzothiadiazole (BTH), an analogue of SA, while mutations in NPR1 reduce the suppression effect due to impaired SA perception. However, the growth suppression effect was reduced with mutations in other NPR1 paralogs (Canet et al., 2010). It has recently demonstrated that NPR1, NPR3, and NPR4 are receptors of SA in Arabidopsis, and the binding of NPR3 and NPR4 to SA is important for their role in NPR1 degradation (Fu et al., 2012; Wu et al., 2012). The double bop1 bop 2 mutant, however, did not show defects in plant resistance, suggesting that BOP proteins might not participate in plant defense systems (Hepworth et al., 2005). Although proteins in the NPR3 subgroup were shown to be involved in plant defense, their functions in plant resistance were contradictory. In Arabidopsis, NPR4 plays a positive role against $P$. syringe (Liu et al., 2005). However, a negative role in regulation of $P R$ genes was later proposed (Zhang et al., 2006). Moreover, VvNPR1.2, which was classified in the NPR3 subgroup (Figure 2) in grape, could not compliment the function of Arabidopsis NPR1 (Le Henanff et al., 2011), suggesting a different function for proteins in the NPR3 subgroup. However, two closely related proteins of VvNPR1.2, GmNPR1-1 and GmNPR1-2 (Figure 2) from soybean, complimented the Arabidopsis npr1-1 mutant and were considered as functional orthologs of Arabidopsis NPR1 (Sandhu et al., 2009). It is possible that different functions have been assigned for evolutionary conserved proteins in different species, but more evidence is needed to obtain a final conclusion.

The redox activity of AtNPR1 is a special posttranslational regulation of this important protein, and both 
$\mathrm{Cys}^{82}$ and $\mathrm{Cys}^{216}$ in the protein sequence are required for its regulation (Mou et al., 2003). The alignment of NPR1 homologs revealed that the $\mathrm{Cys}^{82}$ is conserved among all NPR1 homologs, while the Cys ${ }^{216}$ is conserved only within the NPR1 subgroup (Figure 1B). Indeed, proteins in both the NPR3 and BOP clades, such as AtBOP1, AtNPR4, AtNPR3, MhNPR1, have been localized to the nucleus without presence of reductants (Hepworth et al., 2005; Liu et al., 2005; Zhang et al., 2011; Zhang et al., 2006). In addition to the conserved Cys residues, two regions important for protein interaction with NIMIN proteins are conserved among all NPR1 subclade members including PhaNPR1 (Figure 1B)(Maier et al., 2011). NIMIN proteins are another class of NPR1 interacting proteins, which were first identified with a NPR1 bait, and the interaction plays a regulatory role on activation of NPR1 (Weigel et al., 2001).

The NPR1 homolog from Phalaenopsis aphrodite was classified into the NPR1 subgroup and contains cysteine residues corresponding to $\mathrm{Cys}^{82}$ and $\mathrm{Cys}^{216}$ in AtNPR1 (Figure 1). This result indicates that PhaNPR1 is a bona fide ortholog of AtNPR1, and redox activation might also be a part of its regulation machinery. In addition, transcriptional regulation may also be important for PhaNPR1, as its transcript levels correlated well with PhaPR1 transcript abundance upon SA treatment or challenge with CymMV though PhaNPR1 was only moderately induced by stimuli (Figure 4). We showed that knocking down PhaNPR1 transcription resulted in the reduction of PhaPR1 expression (Figure 5). This result is consistent with our hypothesis that the transcriptional regulation of PhaNPR1 is important for defense in Phalaenopsis and also demonstrates that SAinduced defense may occur through PhaNPR1-mediated pathways.

The PhaNPR1 regulated PR1-like protein, PhaPR1, is much more similar to the Arabidopsis STS14-like protein (AT5G66590), whose function has not been characterized, than to Arabidopsis PR1 (AT2G14610), which is the only SA inducible PR1-like protein in Arabidopsis. However, sequence similarity is not a reliable method for the prediction of protein function; therefore, we identified the promoter region of PhaPR1 and analyzed whether the PhaPR1 can be induced upon SA treatment and virus infection. The promoter region contains many conserved motifs, including an ASF1 motif (important for auxin- and salicylic acid-inducible transcription), an LS10-like motif, and many W-box motifs (potential WRKY transcription factor binding site), which are found in promoter of other PR-1 genes (Table 1). This finding indicates that PhaPR1 might be regulated through machinery similar to that regulating other well-known PR1 proteins. Indeed, subsequent to the induction of PhaNPR1 upon SA treatment and CymMV infection, PhaPR1 was also induced (Figure 4). The expression of PhaPR1 transcripts was reduced when the transcript level of PhaNPR1 was down-regulated (Figure 5). Therefore, PhaPR1 could be a downstream factor in the PhaNPR1-mediated defense pathway. However, the concentrations of CymMV were not altered following PhaNPR1 silencing in CymMV infected plants (Figure 5). $P$. aphrodite infected with CymMV did not show visible symptoms, and still no visible symptoms were observed following PhaNPR1 silencing (data not shown). The role of NPR1 in viral resistance is often contradicted among different plant species. NPR1-dependent pathway is required for proper N-mediated resistance to Tobacco mosaic virus (TMV) in tobacco (Liu et al., 2002). However, in Arabidopsis, resistance to Turnip crinkle virus (TCV) seems required SA but independent to NPR1 because resistance to TCV was not compromised in both npr 1-1 and npr1-5 mutant backgrounds (Kachroo et al., 2000). Furthermore, susceptibility of transgenic rice with ectopic expression of AtNPR1 to Rice yellow mottle virus (RYMV) was increased without alteration on virus concentration (Quilis et al., 2008). It is likely that similar to resistance to TCV in Arabidopsis, resistance to CymMV in P. aphrodite goes through a PhaNPR1-independent pathway. However, we have recently identified a Ring-finger domain containing protein, PhaTF15, and its expression is important for CymMV-induced expression of PhaNPR1 and PhaPR1, and also for resistance to the virus. When expression of PhaTF15 was silenced, the expression of both PhaNPR1 and PhaPR1 were reduced and CymMV accumulated to a high level (Lu et al., 2012). Therefore, a pathway that mediates the expression of PhaNPR1 but not downstream of PhaNPR1 may be important to defense against CymMV in $P$. aphrodite.

\section{Conclusion}

In conclusion, we have identified important components of SAR in orchids and showed that unlike the regulation of Arabidopsis NPR1, the transcriptional regulation of PhaNPR1 plays a pivotal role in the activation of SAR. We also showed that PhaPR1 is a downstream component of PhaNPR1-mediated SAR and have identified the promoter region in the PhaPR1 gene. Additional experiments to identify other elements in the promoter region of PhaPR1 for PhaNPR1-mediated induction are underway.

\section{Additional file}

Additional file 1: Table S1. Differences in the expression of $P R$-1-like genes under pathogen infection or hormone treatment in Arabidopsis thaliana (Data extracted from GENEVESTIGATOR). Table S2. NPR1 homologs used for phylogenetic analysis. Table S3. Primers used in this study.

\section{Abbreviations}

BOP: BLADE-ON-PETIOLE; BTH: benzo-(1,2,3)-thiadiazole-7-carbothioic acid Smethyl ester; CymMV: Cymbidium mosaic virus; GSP: Gene-specific primer; NPR1: NONEXPRESSOR OF PATHOGENESIS-RELATED GENES1; ORF: Open 
reading frame; PR-1: PATHOGENESIS-RELATED GENES -1; RACE: Rapid amplification of CDNA ends; SAR: Systematic acquired resistance.

\section{Competing interests}

The authors declare that they have no competing interests.

\section{Authors' contributions}

$\mathrm{H}-\mathrm{HY}$ and J-CC designed the experiments. H-CL, C-EC, and H-FH did most of the molecular cloning, plant cultivation, chemical treatments, and gene expression analyses. J-CC analyzed the sequences and finished the figures and tables presented in the paper. $\mathrm{H}-\mathrm{HC}$ provided materials. J-CC and $\mathrm{H}-\mathrm{HY}$ drafted the manuscript. All authors read and approved the final manuscript.

\section{Acknowledgments}

This research was funded through grants from National Science Council, Taiwan (grant nos. 98-2321-B-002-019-MY3, 98-2313- B-002-043-MY3 and 101-2321-B-002-048-).

\section{Author details}

${ }^{1}$ Institute of Biotechnology, National Taiwan University, Taipei 106, Taiwan, ROC. ${ }^{2}$ Agricultural Biotechnology Research Center, Academia Sinica, Taipei 106, Taiwan. ${ }^{3}$ Department of Plant Pathology and Microbiology, National Taiwan University, 1, sec 4, Rooselvet Road, Taipei 106, Taiwan. ${ }^{4}$ Research Center for Plant Medicine, National Taiwan University, Taipei 106, Taiwan. ${ }^{5}$ Department of Life Sciences, National Cheng Kung University, Tainan 701, Taiwan. ${ }^{6}$ Institute of Tropical Plant Sciences, National Cheng Kung University, Tainan 701, Taiwan. ${ }^{7}$ Orchid Research Center, National Cheng Kung University, Tainan 701, Taiwan.

\section{Received: 10 June 2013 Accepted: 28 August 2013}

Published: 6 September 2013

\section{References}

Baeuerle PA, Baltimore D (1996) NF-kB: ten years after. Cell 87:13-20

Boyle B, Brisson N (2001) Repression of the defense gene PR-10a by the singlestranded DNA binding protein SEBF. Plant Cell 13:2525-2537

Canet JV, Dobon A, Roig A, Tornero P (2010) Structure-function analysis of npr1 alleles in Arabidopsis reveals a role for its paralogs in the perception of salicylic acid. Plant Cell Environ 33:1911-1922

Cao H, Li X, Dong X (1998) Generation of broad-spectrum disease resistance by overexpression of an essential regulatory gene in systemic acquired resistance. Proc Natl Acad Sci U S A 95:6531-6536

Cao H, Glazebrook J, Clarke JD, Volko S, Dong X (1997) The Arabidopsis NPR1 gene that controls systemic acquired resistance encodes a novel protein containing ankyrin repeats. Cell 88:57-63

Carlson JE, Tulsieram LK, Glaubitz JC, Luk WWK, Kauffeldt C, Rutledge R (1991) Segregation of random amplified DNA markers in $\mathrm{F} 1$ progeny of conifers. Theor Appl Genet 83:194-200

Chern M, Fitzgerald HA, Canlas PE, Navarre DA, Ronald PC (2005) Overexpression of a rice NPR1 homolog leads to constitutive activation of defense response and hypersensitivity to light. Mol Plant Microbe Interact 18:511-520

Chern MS, Fitzgerald HA, Yadav RC, Canlas PE, Dong X, Ronald PC (2001) Evidence for a disease-resistance pathway in rice similar to the NPR1-mediated signaling pathway in Arabidopsis. Plant J 27:101-113

Durrant WE, Dong X (2004) Systemic acquired resistance. Annu Rev Phytopathol 42:185-209

Fu ZQ, Yan S, Saleh A, Wang W, Ruble J, Oka N, Mohan R, Spoel SH, Tada Y, Zheng N, Dong X (2012) NPR3 and NPR4 are receptors for the immune signal salicylic acid in plants. Nature 486:228-232

Glazebrook J, Rogers EE, Ausubel FM (1996) Isolation of Arabidopsis mutants with enhanced disease susceptibility by direct screening. Genetics 143:973-982

Hepworth SR, Zhang Y, McKim S, Li X, Haughn GW (2005) BLADE-ON-PETIOLEdependent signaling controls leaf and floral patterning in Arabidopsis. Plant Cell 17:1434-1448

Kachroo P, Yoshioka K, Shah J, Dooner HK, Klessig DF (2000) Resistance to turnip crinkle virus in Arabidopsis is regulated by two host genes and is salicylic acid dependent but NPR1, ethylene, and jasmonate independent. Plant Cell 12:677-690

Katagiri F, Lam E, Chua NH (1989) Two tobacco DNA-binding proteins with homology to the nuclear factor CREB. Nature 340:727-730
Le Henanff G, Farine S, Kieffer-Mazet F, Miclot AS, Heitz T, Mestre P, Bertsch C, Chong J (2011) Vitis vinifera VvNPR1.1 is the functional ortholog of AtNPR1 and its overexpression in grapevine triggers constitutive activation of $P R$ genes and enhanced resistance to powdery mildew. Planta 234:405-417

Lebel E, Heifetz P, Thorne L, Uknes S, Ryals J, Ward E (1998) Functional analysis of regulatory sequences controlling $P R-1$ gene expression in Arabidopsis. Plant J 16:223-233

Liu G, Holub EB, Alonso JM, Ecker JR, Fobert PR (2005) An Arabidopsis NPR1-like gene, NPR4, is required for disease resistance. Plant J 41:304-318

Liu Y, Schiff M, Marathe R, Dinesh-Kumar SP (2002) Tobacco Rar1, EDS1 and NPR1/NIM1 like genes are required for $\mathrm{N}$-mediated resistance to tobacco mosaic virus. Plant J 30:415-429

Lu HC, Hsieh MH, Chen CE, Chen HH, Wang HI, Yeh HH (2012) A highthroughput virus-induced gene-silencing vector for screening transcription factors in virus-induced plant defense response in orchid. Mol Plant Microbe Interact 25:738-746

Maier F, Zwicker S, Huckelhoven A, Meissner M, Funk J, Pfitzner AJ, Pfitzner UM (2010) NONEXPRESSOR OF PATHOGENESIS-RELATED PROTEINS1 (NPR1) and some NPR1-related proteins are sensitive to salicylic acid. Mol Plant Pathol 12:73-91

Makandar R, Essig JS, Schapaugh MA, Trick HN, Shah J (2006) Genetically engineered resistance to Fusarium head blight in wheat by expression of Arabidopsis NPR1. Mol Plant Microbe Interact 19:123-129

Mitsuhara I, Iwai T, Seo S, Yanagawa Y, Kawahigasi H, Hirose S, Ohkawa Y, Ohashi Y (2008) Characteristic expression of twelve rice PR1 family genes in response to pathogen infection, wounding, and defense-related signal compounds (121/180). Mol Genet Genomics 279:415-427

Mou Z, Fan W, Dong X (2003) Inducers of plant systemic acquired resistance regulate NPR1 function through redox changes. Cell 113:935-944

Niggeweg R, Thurow C, Weigel R, Pfitzner U, Gatz C (2000) Tobacco TGA factors differ with respect to interaction with NPR1, activation potential and DNAbinding properties. Plant Mol Biol 42:775-788

Park HC, Kim ML, Kang YH, Jeon JM, Yoo JH, Kim MC, Park CY, Jeong JC, Moon BC, Lee JH, Yoon HW, Lee SH, Chung WS, Lim CO, Lee SY, Hong JC, Cho MJ (2004) Pathogen- and NaCl-induced expression of the SCaM-4 promoter is mediated in part by a GT-1 box that interacts with a GT-1-like transcription factor. Plant Physiol 135:2150-2161

Pieterse CM, Leon-Reyes A, Van der Ent S, Van Wees SC (2009) Networking by small-molecule hormones in plant immunity. Nat Chem Biol 5:308-316

Quilis J, Penas G, Messeguer J, Brugidou C, San Segundo B (2008) The Arabidopsis AtNPR1 inversely modulates defense responses against fungal, bacterial, or viral pathogens while conferring hypersensitivity to abiotic stresses in transgenic rice. Mol Plant Microbe Interact 21:1215-1231

Rushton PJ, Torres JT, Parniske M, Wernert P, Hahlbrock K, Somssich IE (1996) Interaction of elicitor-induced DNA-binding proteins with elicitor response elements in the promoters of parsley PR1 genes. EMBO J 15:5690-5700

Sandhu D, Tasma IM, Frasch R, Bhattacharyya MK (2009) Systemic acquired resistance in soybean is regulated by two proteins, Orthologous to Arabidopsis NPR1. BMC Plant Biol 9:105

Shi Z, Maximova SN, Liu Y, Verica J, Guiltinan MJ (2010) Functional analysis of the Theobroma cacao NPR1 gene in Arabidopsis. BMC Plant Biol 10:248

Spoel SH, Mou Z, Tada Y, Spivey NW, Genschik P, Dong X (2009) Proteasomemediated turnover of the transcription coactivator NPR1 plays dual roles in regulating plant immunity. Cell 137:860-872

Tian T, Klaassen VA, Soong J, Wisler G, Duffus JE, Falk BW (1996) Generation of CDNAs specific to lettuce infectious yellows closterovirus and other whiteflytransmitted viruses by RT-PCR and degenerate oligonucleotide primers corresponding to the closterovirus gene encoding the heat shock protein 70 homolog. Phytopathology 86:1167-1173

Van Eldik GJ, Wingens M, Ruiter RK, Van Herpen MM, Schrauwen JA, Wullems GJ (1996) Molecular analysis of a pistil-specific gene expressed in the stigma and cortex of Solanum tuberosum. Plant Mol Biol 30:171-176

van Loon LC, Rep M, Pieterse CM (2006) Significance of inducible defense-related proteins in infected plants. Annu Rev Phytopathol 44:135-162

Weigel RR, Bauscher C, Pfitzner AJ, Pfitzner UM (2001) NIMIN-1, NIMIN-2 and NIMIN-3, members of a novel family of proteins from Arabidopsis that interact with NPR1/NIM1, a key regulator of systemic acquired resistance in plants. Plant Mol Biol 46:143-160

Wu Y, Zhang D, Chu JY, Boyle P, Wang Y, Brindle ID, De Luca V, Despres C (2012) The Arabidopsis NPR1 protein is a receptor for the plant defense hormone salicylic acid. Cell Rep 1:639-647 
Yu D, Chen C, Chen Z (2001) Evidence for an important role of WRKY DNA binding proteins in the regulation of NPR1 gene expression. Plant Cell 13:1527-1540

Zhang JY, Qiao YS, Lv D, Gao ZH, Qu SC, Zhang Z (2011) Malus hupehensis NPR1 induces pathogenesis-related protein gene expression in transgenic tobacco. Plant Biol (Stuttg) 1:46-56

Zhang Y, Cheng YT, Qu N, Zhao Q, Bi D, Li X (2006) Negative regulation of defense responses in Arabidopsis by two NPR1 paralogs. Plant J 48:647-656

doi:10.1186/1999-3110-54-31

Cite this article as: Chen et al:: The NPR1 ortholog PhaNPR1 is required for the induction of PhaPR1 in Phalaenopsis aphrodite. Botanical Studies 2013 54:31.

Submit your manuscript to a SpringerOpen ${ }^{\circ}$ journal and benefit from:

- Convenient online submission

- Rigorous peer review

- Immediate publication on acceptance

- Open access: articles freely available online

- High visibility within the field

- Retaining the copyright to your article 